\title{
Identification of TIFY gene family in walnut and analysis of its expression under abiotic stresses
}

Xuejiao Liu ${ }^{1,2 \dagger}$, Feiyan Yu ${ }^{1 \dagger}$, Guiyan Yang ${ }^{1}$, Xiaoqiang Liu ${ }^{3}$ and Shaobing Peng ${ }^{1^{*}}$

\begin{abstract}
Background: Walnuts (Juglans regia L.) are known for their nutrient-rich nuts and are one of the important economic tree species in the world. However, due to global warming and soil salinization, walnuts suffer from various abiotic stresses. TIFY (TIF[F/Y]XG) proteins play an essential role in the growth and development of plants, signal transduction, and stress response in plants. At present, although the TIFY gene family of a number of plants has been identified and studied, how TIFY takes part in stress tolerance remains obscure and many functions of TIFY require further investigation.

Result: In this study, twenty-one TIFY transcription factors were identified in the walnut genome database, and they were divided into four subfamilies (TIFY, JAZ, ZML, and PPD) by bioinformatics analysis. Chromosome location revealed tandem duplication of some genes. Phylogenetic tree analysis showed JrTIFYs were closely related to the TIFY gene family of Arabidopsis thaliana (A. thaliana). QRT-PCR (quantitative real-time PCR) analysis revealed the TIFY genes have different expression patterns in 'Qingxiang' and 'Xiangling' walnut varieties under drought, heat, and salt stress. JAZ subfamily was more expressed in different abiotic stress than other subfamilies. The expressions of JrTIFY14 under heat and salt stress were significantly higher than those under drought stress. However, the expression of JrTIFYs was not significant in 'Xiangling'.
\end{abstract}

Conclusion: This study reveals the TIFY gene family plays an important role in walnuts facing abiotic stresses and provides a theoretical basis for walnut breeding.

Keywords: Walnut, TIFY gene family, Evolution, Phylogeny, Stress expression analysis

\section{Background}

Walnut (Juglans regia L.) is a deciduous tree of the Juglans genus of the Juglandacea family, which is the main cultivated economic tree all over the world. Walnut kernels are one of the most important nuts, which are rich in fat, protein, and various vitamins, and walnut is listed as a priority plant of FAO [1]. As global temperatures

*Correspondence: pshaobing@nwsuaf.edu.cn

${ }^{\dagger}$ Xuejiao Liu and Feiyan Yu contributed equally to this work.

${ }^{1}$ Laboratory of Walnut Research Center, College of Forestry, Northwest

A \& F University, Yangling 712100, Shaanxi, China

Full list of author information is available at the end of the article rise, changes in precipitation systems lead to increased drought stress, resulting in a severe drop in crop yields, especially in Northwest China where walnuts are grown on a large scale $[2,3]$. Due to severe drought and soil salinization in this region, during the growth and development of walnut, it is easy to be subjected to drought and high salt stress $[4,5]$. Therefore, it is of considerable importance for the walnut to breed heat, drought and salt tolerant cultivars and elucidate the mechanisms of abiotic stress responses [6].

The TIFY (TIF[F/Y]XG) gene family is a novel plantspecific gene family recently identified from Arabidopsis thaliana (A. thaliana) [7]. This family has a 
conserved sequence (TIF[F/Y]XG) located in the TIFY domain of about 36 amino acids, which can be divided into four groups: TIFY, JAZ (JASMONATE ZIMDomain), PPD (PEAPOD), and ZML (ZIM-LIKE), by phylogenetic and structural analysis. Four subfamilies of TIFY gene family have different structures. The characteristic of JAZ proteins is the presence of a C-terminal Jas domain, which interacts with the MYC2 proteins to inhibit the jasmonate acid (JA) signaling pathway [7]. PPD protein contains a Jas domain defecting PY-NLS motif (Jasmonate-associate) and a PPD domain, and ZML protein contains a GATA zinc finger and a CCT motif [8-11]. In TIFY domain, glycine is completely conserved and other hydrophobic amino acids are variable. The secondary predicted structure shows that it forms $\alpha-\alpha-\beta$ folded structure [10]. Meloto et al. [12] considered that TIFY domain has a non-negligible role in mediating the interaction between members of TIFY domain and other transcription factors (TFs) [13, 14]. Jas domain, also known as CCT-2 domain, contains the characteristic motif of SLx2Fx2KRx2Rx5PY, which can interact with COI1 and other TFs [8, 12, 15-18].

Plants are subject to biological and abiotic stress inevitably and frequently in their life cycles, which negatively affect their growth, development, and productivity. When plants are under stress, some small signal molecules, such as JA, salicylic acid (SA), ethylene (ET), and abscisic acid (ABA), will help to resist these internal and external stresses. Recently, some studies have identified that the TIFY gene family has multiple regulatory roles in cell signal transduction and plant stress response regulation [19]. Although information about TIFY genes is lacking in most of the plant species, some studies have shown that TIFY gene family plays a key role in plant stress responses, and JAZ protein is one of the most important components of the jasmonate pathway, which has been identified as a transcriptional inhibitor of JA signal $[13,20]$. ZIM is the basis for the formation of homodimers and heterodimers and plays a role in petiole and hypocotyl elongation [18]. Phylogenetic analysis of the GATA gene family in $A$. thaliana revealed that 29 GATA proteins were divided into four subfamilies, three of which encoded ZIM and ZIM-like proteins (ZML proteins). GATA genes participate in the light-responsive, circadian-regulated, and tissue-specific expression patterns [18]. PPDI and PPDII participate in leaf growth coordination [15]. Some studies have found that PPDI proteins can coordinate tissue growth, regulate leaf size and inhibit leaf bending, and the PPDII protein interacts with Gemini al2 protein and shell protein promoter in A. thaliana. Both PPD proteins are closely related to cell cycle arrest [21-23]. Therefore, TIFY gene family plays a significant role in plant growth, development, and stress tolerance.

Although the TIFY genetic family has been studied in the growth and development of some plant species, and its functions have been identified, such as rice (Oryza sativa) [24, 25], soybean (Glycine max) [26], tomato (Lycopersicon esculentum) [27], and Salvia miltiorrhiza (S. miltiorrhiza) [11], few studies have been conducted on the stress resistance of TIFY genes in J. regia. Because of the serious adverse effects of abiotic stresses on the walnut, the study on the TIFY gene family remains necessary. In addition, in our previous research, late-bearing walnuts ('Qingxiang') have higher stress resistance than early-bearing walnuts ('Xiangling'), but the principle is still unknown [28]. In this study, we identified, classified, and made phylogenetic analyses of the TIFY gene family of two walnut species, as well as the levels of TIFY genes in walnut expression in three abiotic stresses with a view to providing a theoretical basis for the evolution of the TIFY gene family and future walnut breeding.

\section{Results}

Genome-wide identification and chromosomal locations of TIFY family genes in J.regia

Twenty-one TIFY genes were identified from J. regia genome by BLAST and named JrTIFY01-JrTIFY21 (Table 1). These genes belonged to four subfamilies, including two members of TIFY subfamily, twelve members of JAZ subfamily, two members of ZML subfamily and two members of PPD subfamily. The molecular weights of TIFY proteins range from $14.46 \mathrm{KDa}$ to 45.68 $\mathrm{KDa}$ and amino acids of them range from 126 to 437. The theoretical PIs of the JrTIFYs are 5.77 to 9.86 . Since JrTIFY10, JrTIFY11, JrTIFY13, and JrTIFY21 have identical gene IDs and protein characters, they are considered to be the same gene. In the following analysis, JrTIFY11, JrTIFY13, and JrTIFY21 are deleted. The results of subcellular location prediction analysis showed that, except for JrTIFY07 and JrTIFY16 located in the chloroplast, the remaining 18 JrTIFYs were located in the nucleus.

According to the gene information, chromosome location analysis was carried out for 18 JrTIFYs (Fig. 1). A total of 18 TIFY genes were located on 11 chromosomes, of which Chr.8 contained three genes (JrTIFY06, JrTIFY10, and JrTIFY17) and Chr.16 contained three genes (JrTIFY09, JrTIFY19, and JrTIFY15). Chr.1, Chr.3, Chr.6, Chr.10, Chr.11, and Chr.15 contained only one TIFY gene.

\section{Phylogenetic analysis of walnut TIFY gene family}

Multiple sequence comparisons revealed that most members of the TIFY gene family contain TIFY and Jas domain (Fig. 2). According to genetic differences, 
Table 1 The TIFY genes in J. regia

\begin{tabular}{|c|c|c|c|c|c|c|c|}
\hline Gene & Accession No & Gene ID & CDS/bp & $\begin{array}{l}\text { Number of } \\
\text { amino acid/aa }\end{array}$ & $\begin{array}{l}\text { Molecular } \\
\text { weight/KDa }\end{array}$ & Theoretical PI & $\begin{array}{l}\text { Subcellular } \\
\text { localization }\end{array}$ \\
\hline JrTIFYO1 & >XP_018823646.1 & LOC108993250 & 1170 & 389 & 41.78 & 8.69 & Nucleus \\
\hline JrTIFYO2 & >XP_018837866.1 & LOC109003977 & 1159 & 386 & 40.77 & 8.85 & Nucleus \\
\hline JrTIFYO3 & >XP_018845750.1 & LOC109009626 & 1299 & 432 & 45.16 & 8.76 & Nucleus \\
\hline JrTIFYO4 & >XP_018839218.1 & LOC109004963 & 858 & 285 & 31.27 & 9.3 & Nucleus \\
\hline JrTIFY05 & >XP_018839402.1 & LOC109005076 & 1026 & 341 & 37.19 & 8.54 & Nucleus \\
\hline JrTIFYO6 & >XP_018834762.1 & LOC109001793 & 1314 & 437 & 45.68 & 9.14 & Nucleus \\
\hline JrTIFYOT & >XP_018835038.1 & LOC109001963 & 798 & 265 & 29.21 & 8.98 & Chloroplast \\
\hline JrTIFYO8 & >XP_018818614.1 & LOC108989462 & 984 & 327 & 35.93 & 9.12 & Nucleus \\
\hline JrTIFYO9 & >XP_018826622.1 & LOC108995501 & 1158 & 385 & 40.81 & 8.62 & Nucleus \\
\hline JrTIFY10 & >XP_018817541.2 & LOC108988673 & 918 & 305 & 32.76 & 5.77 & Nucleus \\
\hline JrTIFY11 & >XP_018817541.2 & LOC108988673 & 918 & 305 & 32.76 & 5.77 & Nucleus \\
\hline JrTIFY12 & >XP_018814737.1 & LOC108986549 & 825 & 274 & 29.51 & 9 & Nucleus \\
\hline JrTIFY13 & >XP_018817541.2 & LOC108988673 & 918 & 305 & 32.76 & 5.77 & Nucleus \\
\hline JrTIFY14 & >XP_018840946.1 & LOC109006194 & 1152 & 383 & 41.49 & 9.45 & Nucleus \\
\hline JrTIFY15 & >XP_018809159.1 & LOC108982287 & 708 & 235 & 25.93 & 8.42 & Nucleus \\
\hline JrTIFY16 & >XP_018805458.1 & LOC108979265 & 819 & 272 & 29.46 & 9.13 & Chloroplast \\
\hline JrTIFY17 & >XP_018817541.2 & LOC121263054 & 918 & 305 & 32.76 & 5.77 & Nucleus \\
\hline JrTIFY18 & >XP_018810390.1 & LOC108983260 & 429 & 126 & 14.46 & 9.86 & Nucleus \\
\hline JrTIFY19 & >XP_018806456.1 & LOC108980095 & 579 & 192 & 20.85 & 8.79 & Nucleus \\
\hline JrTIFY2O & >XP_018859193.1 & LOC109021098 & 579 & 192 & 21 & 9.54 & Nucleus \\
\hline JrTIFY21 & >XP_018817541.2 & LOC108988673 & 918 & 305 & 32.76 & 5.77 & Nucleus \\
\hline
\end{tabular}

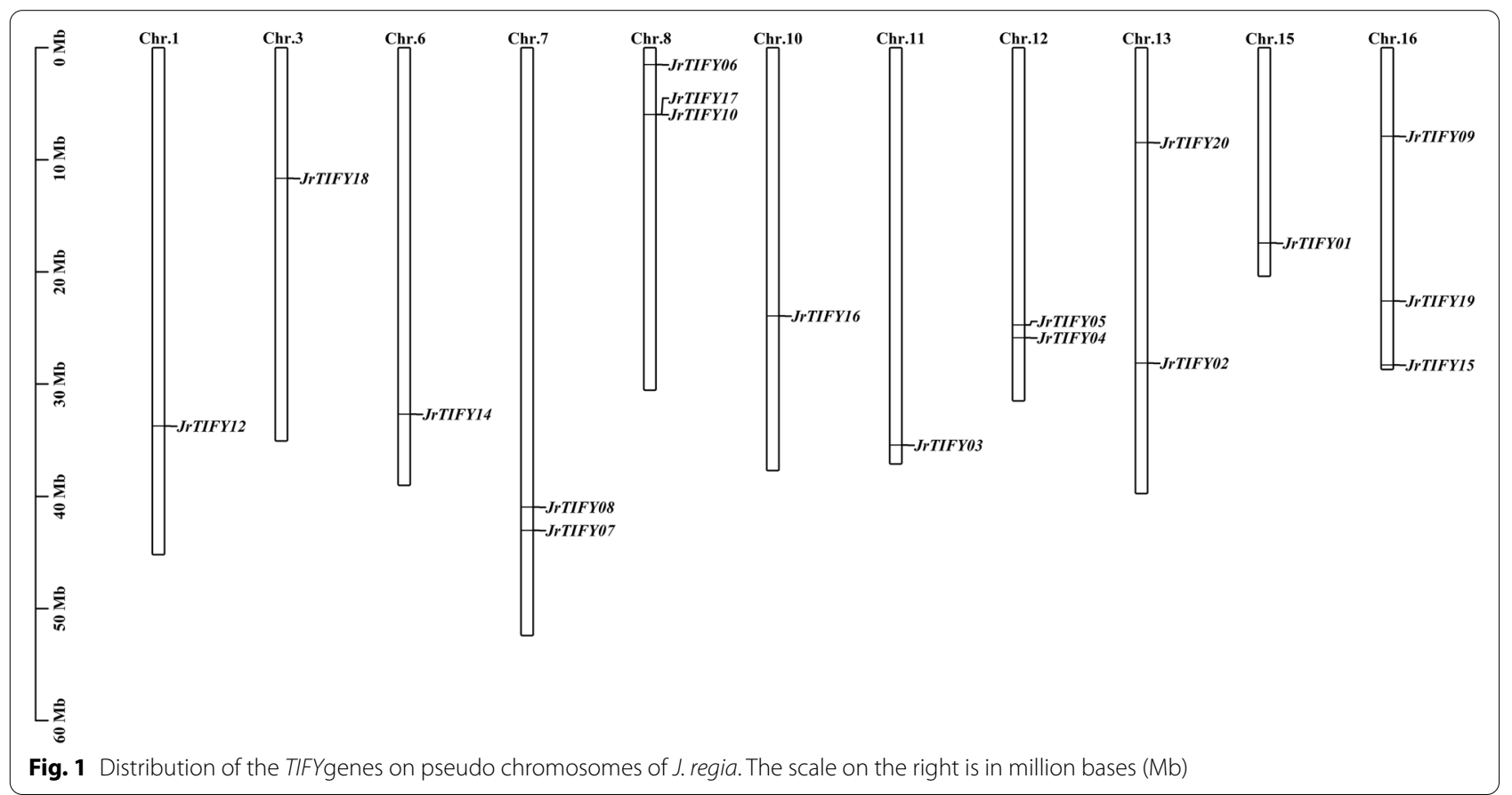

a neighbor-joining phylogenetic tree with TIFY genes of J. regia, A. thaliana and Populus trichocarpa ( $p$. trichocarpa) was built (Fig. 3). A total of 50 genes of
J. regia, A. thaliana, and $p$. trichocarpa were divided into eight branches: proteins containing Jas conservative domain structure were divided into five groups, 


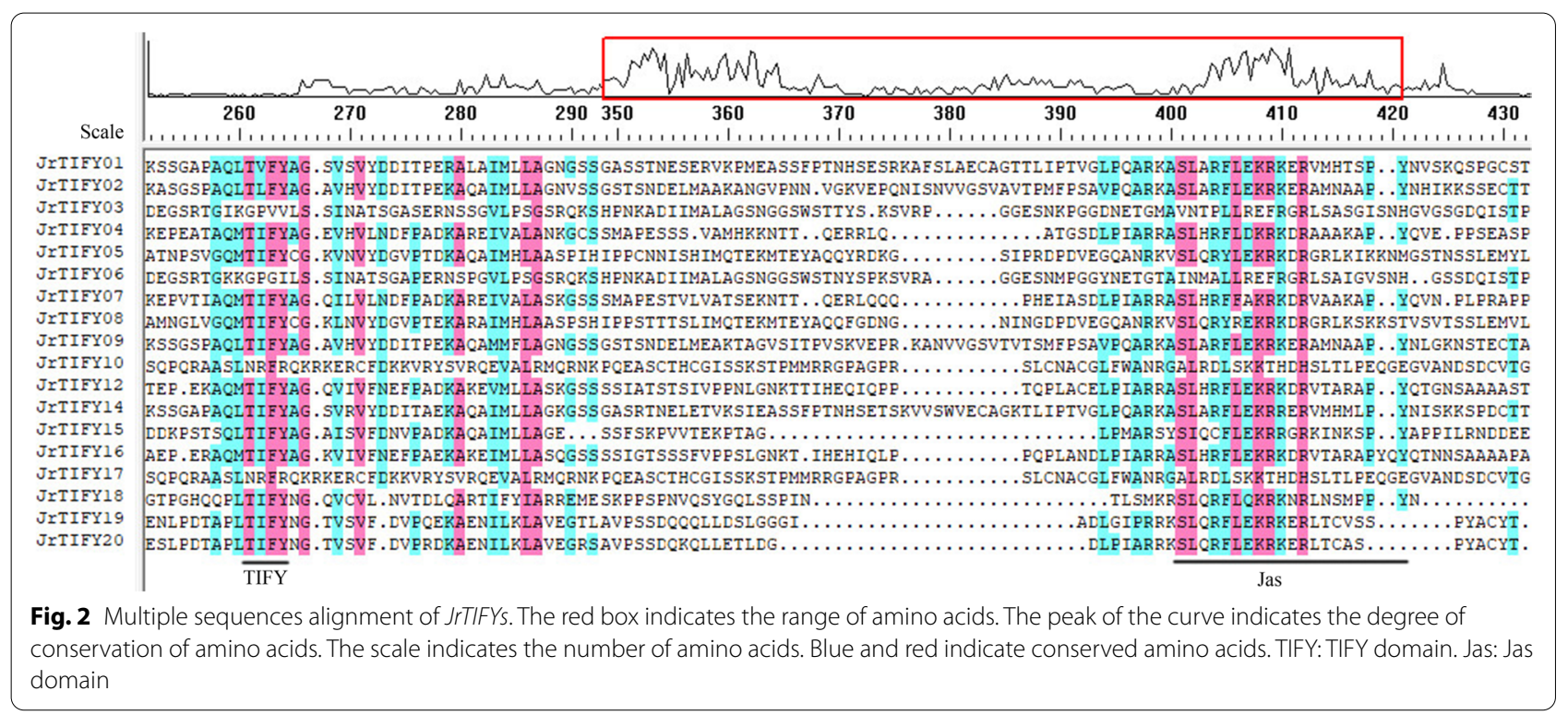

named groups I-V for JAZ family, including $12 \mathrm{JrTI}$ $F Y \mathrm{~s}, 11$ AtTIFYs, and 10 PtTIFYs. ZML/ZIM proteins contained 2 JrTIFYs, 1 AtTIFYs, and 5 PtTIFYs. TIFY protein contained 2 JrTIFYs and 1 PtTIFYs. PPD protein contained 2 JrTIFYs and 2 AtTIFYs. The JAZ subfamily contained the most TIFY genes, and these genes varied widely, showing that the evolution of JAZ subfamily was more extensive in evolutionary history. Of the five branches of the JAZ subfamily, group I, II, III and group V contained two JrTIFYs, respectively, group IV contained four JrTIFYs. Moreover, the results showed that there were 5 pairs of orthologous genes (JrTIFY19 and JrTIFY20, JrTIFY04 and JrTIFY07, JrTIFY14 and JrTIFY01, JrTIFYO8 and JrTIFY05, JrTIFY10 and JrTIFY17) and 5 pairs of paralogous genes (JrTIFY16 and AT1G74950.1, JrTIFY12 and AT1G19180.1, JrTIFYO2 and AT3G17860.1, JrTIFY15 and AT4G32570.1, JrTIFY18 and Potir.011G083900.1).

\section{Conservative motif distribution of walnut TIFY gene family} According to the conserved regions, MEME motif analysis identified a total of 15 motifs from $18 \mathrm{JrTIFYS}$ (Fig. 4 and Table 2). Each JrTIFY contained 2-8 motifs. Since TIFY subfamily contains the TIFY domain, motif2 (PATAQLTIFYAGQVHVFD) was annotated as the TIFY domain, and motif1 (LPIARKASLQRFLEKRKERVT) was annotated as Jas domain, which was contained in genes of the JAZ, TIFY and PPD subfamily, but not in the ZIM subfamily. JrTIFYO3 and JrTIFY06 contained motif12 (NHLEGIPFYGPRSDISGPEISNRIVGSKRSNPDSAFLGSFRDGIINLDHD) and motif14 (WEWPASMNVGPAVKYPPRGGQFGPILHQVPSNRFRDGTAGPSCISQSAAD), none of these in other
JrTIFYs, which were annotated as GATA zinc-finger and CCT structure. JrTIFY05 and JrTIFYO8 contained motif15 (ILDKPLNQLTEEDIAQLTREDCRKFLKDK),

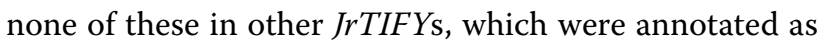
PPD domain. JrTIFY10 and JrTIFY17 have seven same motifs. JrTIFY01, JrTIFY02, JrTIFY09, and JrTIFY14 contained the same motifs. JrTIFY18 contained the least domain, only having motif1 and motif 2 . Moreover, differences in exon-intron structure play an important role in gene evolution. In order to study the diversity of walnut TIFY genes structure, the exon-intron of each TIFY was investigated (Fig. 5). These results showed that most JrTIFYs contained more than three exons. JrTIFYO5 and JrTIFY08 contained the highest number of exons with eight, and JrTIFY10 and JrTIFY18 had the fewest number of exons with three. The amino acids motif7, motif9 and motif15 were highly conserved, revealing JrTIFYs were highly conservative (Fig. S1).

\section{Cis-acting element analysis of walnut TIFY gene family}

In order to study the potential biological functions of the JrTIFYs, the genomic sequence of 2000 bp upstream of the JrTIFYs was extracted as a hypothetical promoter sequence for cis-acting element analysis (Fig. 6). The results showed that there were four types of cis-elements in the 18 members, which were plant photoreaction elements, hormone response elements, plant growth and development elements, and stress response elements (Fig. S3). In addition to JrTIFY03, other JrTIFYs all contained ABA response elements, which were related to the opening and closing of stomata, osmotic stress and stress resistance [29]. In addition to JrTIFY18, other JrTIFYs all contained MeJA response elements, which stimulated the 


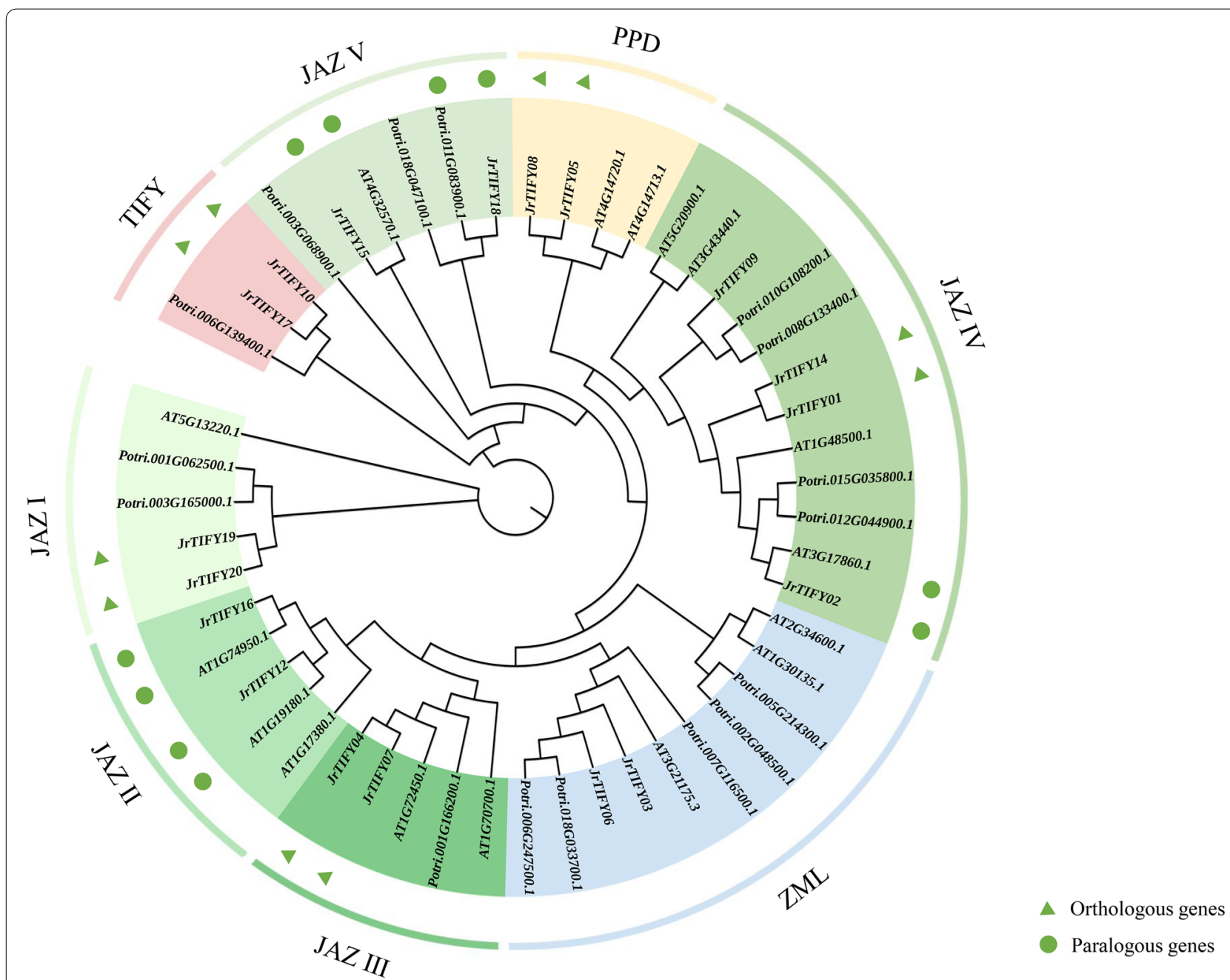

Fig. 3 Phylogenetic tree of TIFY gene in P. trichocarpa (Potri.), A. thaliana (AT) and J. regia (Jr). Orthologous and paralogous genes were indicated by a triangle and roundness, respectively

expression of defensive plant genes [30]. More than half of the genes contained gibberellin response elements, SA response elements and auxin response elements. In addition, some genes contained elements related to stress response such as drought, anaerobic induction, low temperature, and wound response. JrTIFYO1 and JrTIFY08 contained flavonoid synthesis elements, which were also closely related to stress response [31].

\section{Expression characteristics of the TIFY gene family under abiotic stresses}

The expression characteristics of eighteen JrTIFYs in leaves of 'Qingxiang' and 'Xiangling' walnut varieties under drought, heat, and salt stresses were shown in Fig. 7.

For 'Qingxiang' (Fig. 7a). Under three stresses, the expression of some JrTIFYs was high, such as JrTIFY10, JrTIFY01, JrTIFY03, and JrTIFY19, and the expression of JrTIFY14 was higher in heat stress and salt stress than in drought stress. However, the expression of some $\mathrm{JrTI}$ FYs was low, such as JrTIFY18, JrTIFY08, and JrTIFY15. Under drought stress, JrTIFY10, JrTIFY01, JrTIFY03, JrTIFY05, and JrTIFYO6 reached the trough value in $18 \mathrm{~d}$ (D-18). While JrTIFY19 showed the high expression in $18 \mathrm{~d}$ (D-18) and $20 \mathrm{~d}$ (D-20). JrTIFY10 showed the highest expression in $22 \mathrm{~d}$ (D-22), and its expression decreased after rehydration (R). The expression of JrTIFYO1 was the highest at $7 \mathrm{~d}$ (D-7). Under heat stress, JrTIFY08, JrTIFY15, and JrTIFY18 showed low expressions throughout the whole process of heat stress, while JrTIFY14 showed a high expression throughout the whole process with the highest content at $8 \mathrm{~h}(\mathrm{H}-8)$. The expression of JrTIFYO1 was the highest at $2 \mathrm{~h}(\mathrm{H}-2)$. Under salt stress, the expressions of JrTIFY09, JrTIFY18, JrTIFY08, and JrTIFY15 were low, while the expressions of JrTIFY14, JrTIFY10, and JrTIFY01 reached peak value 


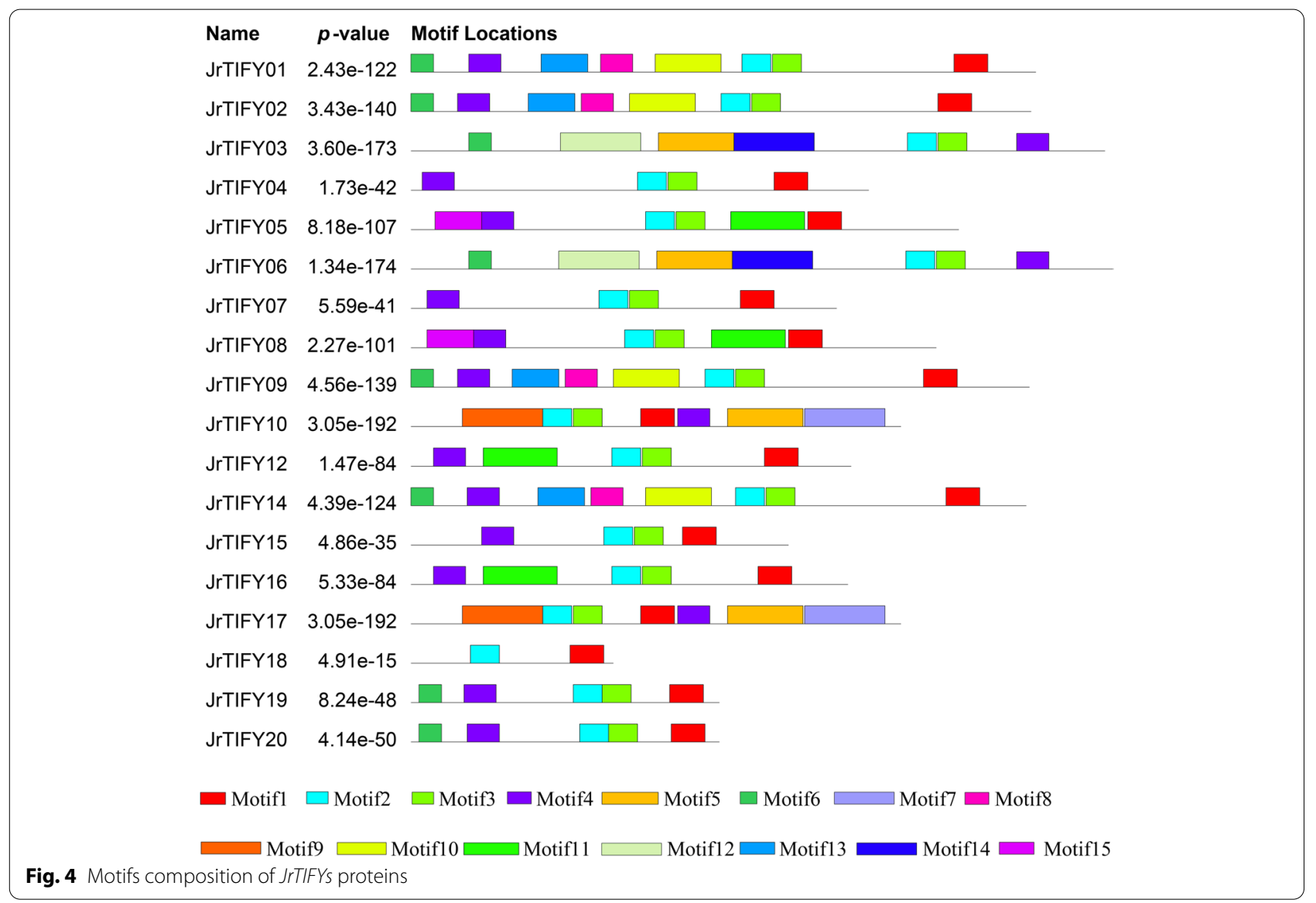

Table 2 Motif sequences identified by MEME tool

\begin{tabular}{lll}
\hline Motif & Number of amino acids & Best possible match \\
\hline Motif1 & 21 & LPIARKASLQRFLEKRKERVT \\
Motif2 & 18 & PATAQLTIFYAGQVHVFD \\
Motif3 & 18 & VPPDKAQAIMLLAGKGSS \\
Motif4 & 20 & GMQRSFRNKCSAJSQYNKEK \\
Motif5 & 47 & RWGAGGESGRDDNPDEVSFTHCPJRPKSAPLVRRPPAGPRSDANVSG \\
Motif6 & 14 & MERDFLGLSKKTDS \\
Motif7 & 50 & FWANRGALRDLSKKTHDHSLTLPEQGEGVANDSDCVTGIHTNNNLVTYSN \\
Motif8 & 20 & YPPQQHDAHSVHRPHDVKIF \\
Motif9 & 50 & IDNSLIRYEAHSIDDAAGSVGGVDDVTADAVYSHGGSDGGASEMVIQRH \\
Motif10 & 41 & GGTPFFKNHFATGQNLVGSTIKPPPLGGIPPVAPHPSTPSI \\
Motif11 & CNJZGNGDKDHLIPPAPTMSLFPQTEKLTDYAQQNVDAGQNPKPPD \\
Motif12 & 46 & NHLEGIPFYGPRSDISGPEISNRIVGSKRSNPDSAFLGSFRDGIINLDHD \\
Motif13 & 50 & TADVFDTNKKPYSGEIQKNLNHDKQGGTH \\
Motif14 & 29 & WEWPASMNVGPAVKYPPRGGFGPILHQVPSNRFRDGTAGPSCISQSAAD \\
Motif15 & 50 & ILDKPLNQLTEEDIAQLTREDCRKFLKDK \\
\hline
\end{tabular}




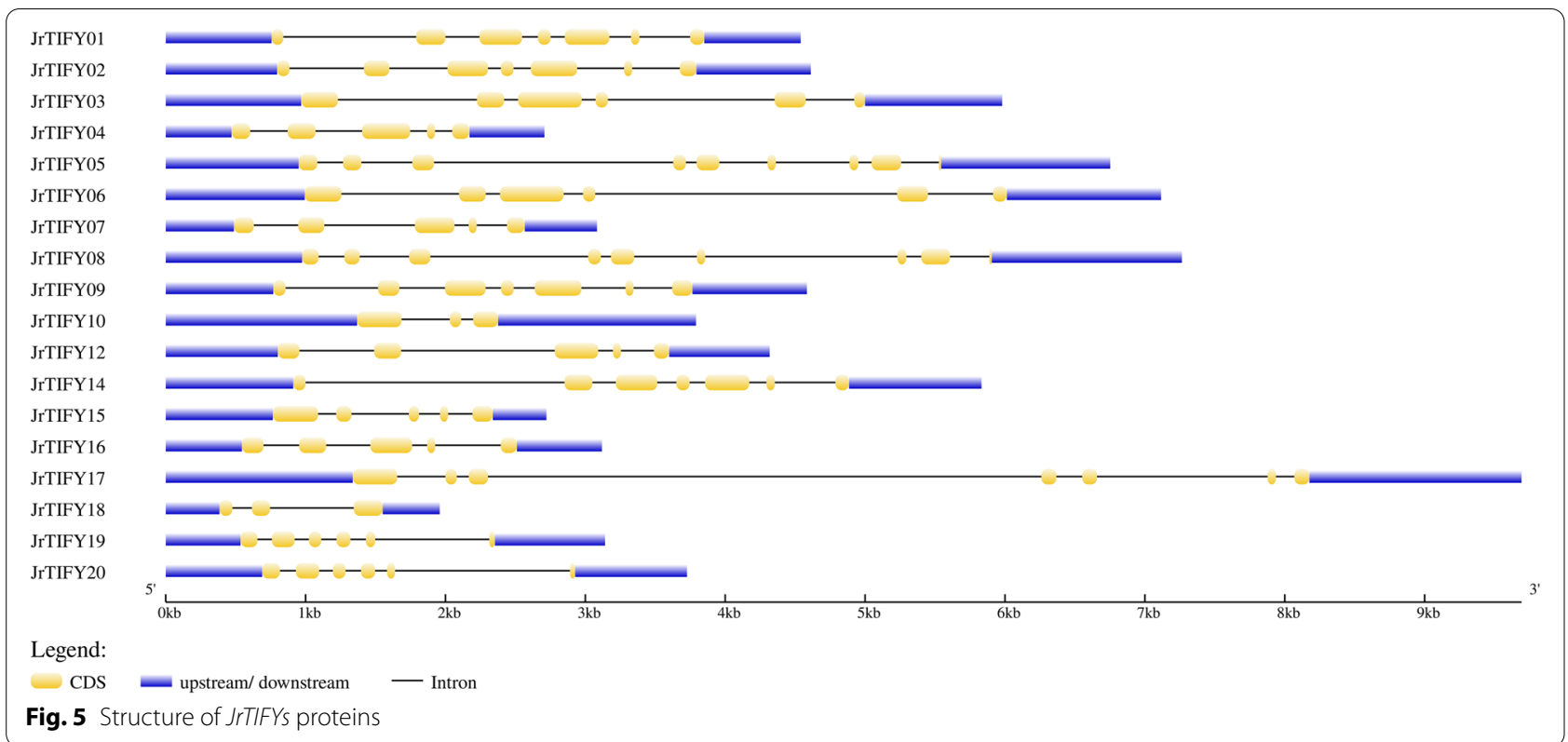

at $24 \mathrm{~h}$ (S-24). JrTIFY19 was particularly prominent with a increase in content from 6 to $24 \mathrm{~h}$, but decreased at $72 \mathrm{~h}(\mathrm{~S}-72)$.

For 'Xiangling' (Fig. 7b). Under drought, heat, and salt stress, the expression of JrTIFY07 and JrTIFY20 was high, and the expression of JrTIFY14 was similar to that in 'Qingxiang', but the overall expressions of most JrTIFYs were very low with almost no expression of JrTIFY08 and JrTIFY15. Under drought stress, the expression level of JrTIFY07 gradually decreased with time (D-7 D-22), but increased after rehydration (R). Under heat stress, JrTIFY14 showed the highest expression in $2 \mathrm{~h}(\mathrm{H}-2)$, and JrTIFY03, JrTIFY05, JrTIFY06, JrTIFY01, and JrTIFYO2 reached the peak in $8 \mathrm{~h}(\mathrm{H}-8)$. Under salt stress, the expressions of JrTIFY07 and JrTIFY20 showed an increasing trend over time, but JrTIFY07 showed a decreasing trend $(\mathrm{S}-6 \sim \mathrm{S}-72)$. It is worth mentioning that JrTIFY07 and JrTIFY20 were highly expressed in 'Xiangling', but low in 'Qingxiang', while JrTIFY01 and JrTIFY03 were opposite under three stresses.

\section{Discussion}

\section{The walnut TIFY genes display diverse characters}

With the development of genomics, more and more plant genomes have been analyzed and the TIFY gene family as a specific TF has been identified in more plants. A total of $18 \mathrm{JrTIFY}$ s were identified, and phylogenetic trees were established with sixteen genes from A. thaliana and sixteen genes from $P$. trichocarpa (Fig. 3). There were four paralogous gene JrTIFYs between the walnut and A. thaliana (JrTIFY16 and AT1G74950.1, JrTIFY12 and AT1G19180.1, JrTIFY02 and AT3G17860.1, JrTIFY15 and
AT4G32570.1), indicating the relationship between the two plant species was closely related during the evolution [10]. Moreover, there was a pair of JrTIFYs (JrTIFY18 and Potri.011G083900.1) with similarity in walnut and P. trichocarpa. JrTIFYO3 and JrTIFYO6 belonged to the ZML subfamily and closely related to the ZML subfamily of AT3G21175.3 (AtZML01) and Potri.007G116500.1 (PtZML02). Wen et al. [32] think that two genes of the ZML subfamily (CsTIFY3 and CsTIFY12) also have a very high similarity, and Zhao et al. [33] find that several pairs of homologous genes of the ZML subfamily have a very high similarity, indicating that the ZML subfamily may have evolved without variation.

The online tool MEME was used to search for fifteen conservative structures of JrTIFY protein (Fig. 4). Among them, motif1 was predicted to be Jas domain, motif 2 to be TIFY structure, and motif12 and motif14 to be GATA zinc-finger structure and CCT structure. JrTIFY19 and JrTIFY20 were the same motifs and the located in the closest phylogenetic branches, which belonged to the JAZ subfamily. In Zhao's [33] studies, PviTIFFY10 and PviTIFFY11 are located in the closest phylogenetic branches, containing the same motifs, namely $\mathrm{N}$-terminal, TIFY, Jas, belong to the JAZ subfamily. Ye et al. [25] think that just OsTIFY10 and OsTIFY11 of the JAZ subfamily in rice have NT motif at N-terminal. These results indicated that the special structure of genes during the evolution of plant species will make the species unique. Moreover, the chromosome location showed that JrTIFY10 and JrTIFY17 were at the same position on the same chromosome, indicating that there may be redundancy or tandem duplications, which was consistent 


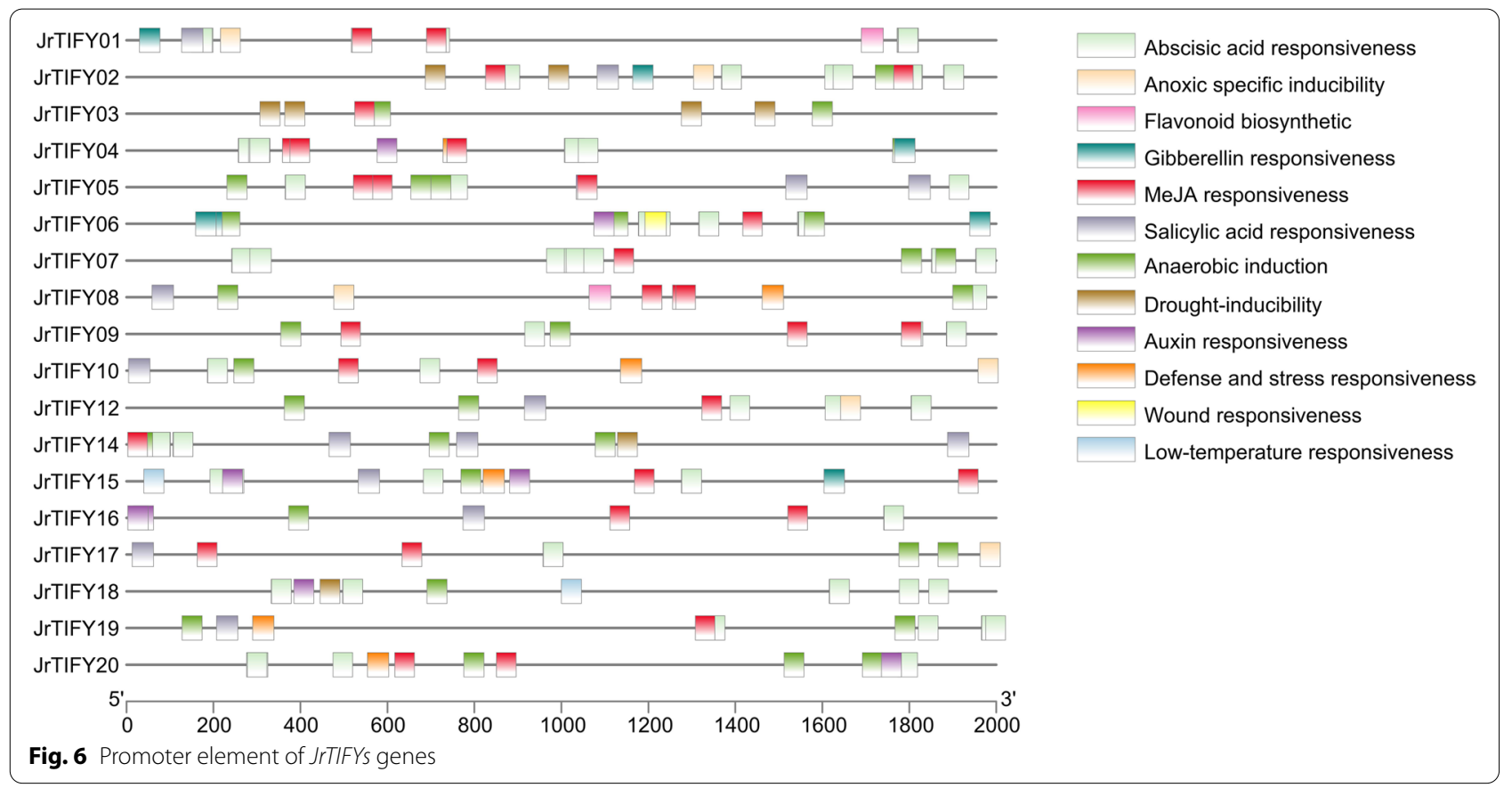

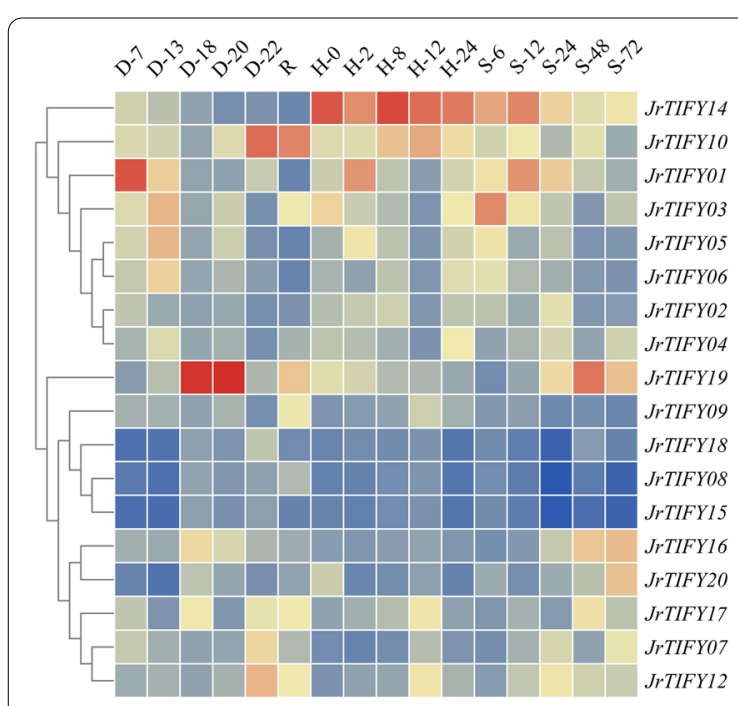

(a)

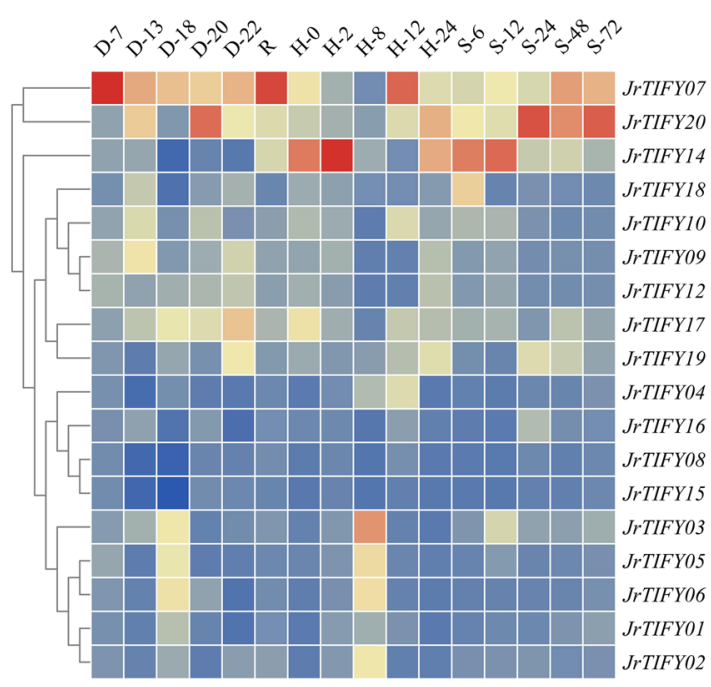

(b)

Fig. 7 Stress expression profile of JrTIFYS. D-7 / D-13 / D-18 / D-20 / D-22: 7, 13, 18, 20, $22 \mathrm{~h}$ after drought treatment. R: rehydration after drought treatment. $\mathrm{H}-0 / \mathrm{H}-2 / \mathrm{H}-8 / \mathrm{H}-12 / \mathrm{H}-24: 0,2,8,12$ and $24 \mathrm{~h}$ after heat treatment. S-6 / S-12 / S-24 / S-48 / S-72: 6, 12, 24, 48 and $72 \mathrm{~h}$ after salt treatment. a Three abiotic stresses of 'Qingxiang'; b Three abiotic stresses of 'Xiangling'

throughout the study of Zhang et al. [34]. The study of Zhang et al. shows that VvJAZ5, VvJAZ6, VvJAZ7 and $V v J A Z 8$ are tandem duplications in grapes, which may be the main reason for the expansion of the plant genes' family.

Among the JAZ subfamily, twelve JrTIFYs contained Jas domain, which was the most numerous subfamily, similar to the TIFY gene family of other species such as rice [24, 25], S. miltiorrhiza [11] and soybean [26], speculating that the JAZ subfamily may have greater potential in regulating plant growth and development [10]. Previous studies have shown $[7,35]$ that the JAZ subfamily is an important regulatory factor that controls the initiation of JA by responding to JA stimulation. The JAZ protein 
usually acts as an inhibitor in the JA signaling pathway, releasing targeted TFs [36]. Through the analysis of promoter elements, we found that almost JrTIFYs contained MeJA response elementsm. The research of Lotfi et al. [37] shows that JA is the key hormone for sensitive genotypes to resist stress. It was speculated that TIFY gene family may participate in walnut resistance through JA pathway. Meanwhile, the JAZ subfamily also plays an important role in ABA signaling pathway regulation and plant physiological processes, such as photosynthesis [11, 32, 38]. The study of Zhao et al. [26] suggests that the JAZ subfamily in soybean may be involved in JA signaling pathway to resist saline alkali stress and GsJAZ2 overexpression promotes soybean growth in alkaline environment. The JAZ subfamily of $S$. miltiorrhiza negatively regulates the biosynthesis of salvianolic acid and tanshinone [11]. The research of Zhao et al. [33] shows that the PviTIFY10 of the JAZ subfamily could be induced by ABA in switchgrass (Panicum virgatum). Therefore, it can be speculated that the JAZ subfamily has a certain role in different physiological processes in the process of walnut growth.

\section{JrTIFYs expression patterns to abiotic stress implied multiple roles}

Under the three stresses, most JrTIFYs showed a higher expression level of 'Qingxiang' than 'Xiangling' (Fig. 7), showing that 'Qingxiang' may be more resistant than 'Xiangling', which is also in line with previous research [28]. The study of An et al. [39] also prove that overexpression of apple (Malus domestica) MdJAZ2 improves the resistance of $A$. thaliana to $\mathrm{NaCl}$ and drought treatments. Similarly, the overexpression of OsTIFY11a/OsJAZ9 in rice significantly enhanced tolerance to salt and dehydration stress [35]. Gene expression profiles of JrTIFYs were different under drought, heat, and salt stress. For example, the response of JrTIFY14 to salt stress and heat stress was stronger than that of drought stress, indicating that JrTIFY14 may have a negative regulatory effect on drought stress. Xie et al. [40] carries out that all homologous genes of TaJAZ3 are highly activated by heat, but are significantly inhibited by drought in wheat (Triticum aestivum). Similarly, He et al. [41] think TIFY11b, TIFY10a, TIFY3, TIFY5a/b, and TIFY6 of JAZ subfamily are induced by cold acclimation but not by the chilling treatment in Brassica napus. The expression profiles of the same $\operatorname{JrTIFY}$ were also different under the same stress of different walnut varieties. The expression of JrTIFY01 in 'Qingxiang' first decreased and then increased under drought stress, while the expression level first increased and then decreased in 'Xiangling. In addition, under the same stress, the JrTIFYs had different stress time to reach the maximum expression. For example, under salt stress, the expression level of JrTIFY20 reached its peak in 'Qingxiang' in $72 \mathrm{~h}$, and reached its peak in 'Xiangling' in $24 \mathrm{~h}$. This phenomenon was consistent with Wang's [42] research, indicating that different genes had the different response time to stress.

The members of JrTIFYs had different response mechanisms under different stresses of the same species or under the same stress of different species. The expression of JrTIFY10, JrTIFY01, and JrTIFYO3 was significantly different in 'Qingxiang' and 'Xiangling', they were more significant in 'Qingxiang', but hardly expressed in 'Xiangling', but JrTIFYO7 and JrTIFY2O were opposite, speculating that the resistance of early and late walnuts to abiotic stresses may be related to these genes [43]. Under the same stress, the genes that came from the JAZ subfamily had the most significant expression levels in varieties. For 'Qingxiang', the expression of JrTIFY19 had the greatest change under salt stress, while for 'Xiangling', the expression of JrTIFY2O had the greatest change. Liu et al. [44] believes that TIFY genes have different responses to different hormone treatments, and the expressions of genes belonging to the JAZ subfamily increases after MeJA treatment, but decreases after SA/ET treatment, revealing that JAZ plays an important role in the response of plants to abiotic stress.

In the four subfamilies of the TIFY gene family, the expression amount of the JAZ subfamily changed more significantly under different abiotic stresses. The study of Heidari et al. shows that the JAZ subfamily responds more strongly to abiotic stress than other subfamily in maize (Zea mays) and tomato [19]. Our research results led to similar conclusions, for instance, JrTIFY14, belonging to the fourth branch of the JAZ subfamily, was highly expressed under heat and salt stress, indicating that JrTIFY14 may have a certain effect on walnuts in response to abiotic stress, which was consistent with Wang's [10] research results. Wang believs that the JAZ subfamily of poplar (populus) has a high expression level in different tissues under different stresses, and PtJAZ8 is significantly expressed in all tissues. PtJAZ6, PtJAZ7, and PtJAZ12 were highly expressed in the phloem, xylem, and leaf tissues. Zhang et al. [38] believes that JA induces all CsJAZs in tea plants, and these genes show different responses to mechanical injury and bacterial infection. However, under the three stresses of walnut, the basically unexpressed JrTIFY15 also belonged to the JAZ subfamily, while in Wang's study [8], PtJAZ1 shows low expression in different tissues of poplar, suggesting that different $J A Z$ genes possess distinct functions, and JAZ subfamily may play an obvious role in resistance to abiotic stresses in the plant. 


\section{Conclusions}

In this study, a total of $18 \operatorname{JrTIFYs}$ were identified and their chromosomal distribution, conserved domain structure, and phylogenetic tree were analyzed. The study concluded that JrTIFYs were highly conserved and were closely related to the TIFY gene family of $A$. thaliana. Under the stress of drought, heat, and salt, the expressions of different genes were various, in particular, the JrTIFY14 of JAZ subfamily positive feedback regulated heat and salt stress in both varieties, but negative feedback regulated drought stress. JAZ subfamily played an important role in plant growth and development. However, different walnuts had different responses to abiotic stress. The expression of JrTIFYs in 'Qingxiang' and 'Xiangling' revealed that JrTIFYs may play a key regulatory role in the abiotic stress on walnuts. This study provides a theoretical basis for further study on the regulatory mechanism of TIFY gene family and the genetic breeding of walnut.

\section{Materials and methods \\ Plant materials and treatments Seedling preparation}

Two walnut varieties 'Qingxiang' (late-bearing walnuts) and 'Xiangling' (early-bearing walnuts), two-yearold seedlings, were treated with drought, salt, and heat stress. Under the conditions of $22 \pm 2{ }^{\circ} \mathrm{C}, 70 \pm 5 \%$ relative humidity and $14 \mathrm{~h}$ light/10 h dark, new branches scions of walnut grafted into the walnut rootstocks (J. regia) which were planted in the mixed substrate of turf peat and sand $(2: 1 \mathrm{v} / \mathrm{v})$ in the greenhouse located in Northwest A\&F University (Yangling, Shaanxi province, China). Walnut seedlings with a height of $55-65 \mathrm{~cm}$ had similar biomass, and walnut leaves were collected as experimental samples, which were frozen in liquid nitrogen immediately and stored in $-80^{\circ} \mathrm{C}$ refrigerator for further experiments [28].

\section{Drought treatment}

Firstly, fifteen 'Qingxiang' and fifteen 'Xiangling' walnuts were transplanted into the prepared flowerpots with the same conditions, and the soil moisture content of each pot was maintained at $60 \%$ for seven days. When the soil water content reached $60 \%$ (7 d), 40\% (13 d), 20\% (18 d), $10 \%(20 \mathrm{~d})$, and $5 \%(22 \mathrm{~d})$ without watering, the leaves of nine plants of each variety were collected for three repeated groups. Three plants of the two varieties continued to be re-watered after $22 \mathrm{~d}$ to make the soil moisture content reach $60 \%$ and kept for 3 days, collecting leaves. The remaining three plants of the two varieties were watered as control $[28,45]$.

\section{Heat treatment}

Twelve 'Qingxiang' and twelve 'Xiangling' walnut seedlings were subjected to heat stress at $42^{\circ} \mathrm{C}$ for $2 \mathrm{~h}$. Leaves were collected respectively for three repeated groups at $0,2,8,12$, and $24 \mathrm{~h}$ after treatment. In addition, three seedlings of each walnut variety were cultured at $25^{\circ} \mathrm{C}$ as control [28].

\section{Salt treatment}

A total of twenty-four plants (twelve plants of each variety) were treated with $1 / 2$ Hoagland with $300 \mathrm{mM} \mathrm{NaCl}$. The leaves were collected at 6, 12, 24, 48, and $72 \mathrm{~h}$ after treatment respectively for three repeated groups. In addition, three seedlings of each walnut variety were watered with $\mathrm{NaCl}$-free $1 / 2$ Hoagland solution as control $[28,46]$.

\section{Identification and chromosomal locations of the walnut TIFY gene family}

The conserved domain information of TIFY genome was downloaded from Pfam website (http://pfam. sanger.ac.uk), and the chromosome position of TIFY gene family in the walnut genome (J. regia) was obtained on NCBI website (https://doi.org/10.1093/ gigascience/giaa050) [47]. The TIFY genes sequence information of walnut was downloaded from iTAK website (http://itak.feilab.net/cgi-bin/itak/db_family_ gene_list.cgi?acc=Tify\&plant=51240). The TIFY gene family of walnut, $A$. thaliana and $P$. trichocarpa was searched by blast on NCBI website, and the coding regions' gene sequences of TIFYs were obtained by UltraEdit software [48, 49]. The chromosome location, sequence ID, protein sequence, amino acid number, protein molecular weight $(\mathrm{kDa})$, and protein isoelectric point (PI) were downloaded from ExPASY website (http://web.expasy.org/protparam/). The predictive analysis of the subcellular localization of walnut TIFY protein was performed by using the WoLF PSORT (https://www.genscript.com/wolf-psort.html) [50].

\section{Multiple alignments and phylogenetic analyses}

Phylogenetic reconstruction is an important part of genomics. DANMAN software was used for multiple comparison of walnut TIFY protein. Clustal $\times 2.1$ software was used to multi-aligned amino acid sequences of TIFY proteins in walnut [51]. The phylogenetic tree was established by using MEGA6.0 software with a neighborhood connection method for the protein sequences of TIFY in walnut, A. thaliana and $p$. trichocarpa (bootstrap $=1000)$ [52]. The amino acid sequence with FASTA format was input into the online tool MEME (Multiple 
Em for Motif Elicitation) (http://meme-suite.org/tools/ meme) to predict the structures, and the conservative motif searched was 15 , and the others are default values [53]. The $2000 \mathrm{bp}$ upstream of the start codon of the walnut TIFY gene family was extracted and uploaded to PlantCARE (http://bioinformatics.psb.ugent.be/webto ols/plantcare/html/) by using TBtools software to predict and analyze the cis-acting elements in the promoter region [50].

\section{qRT-PCR analysis}

Total RNA was extracted from walnut leaves of 'Qingxiang' and 'Xiangling' varieties by CTAB method [54]. Because four of the TIFY genes (JrTIFY10, JrTIFY11,

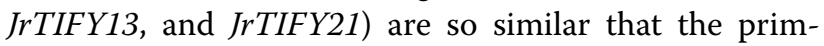
ers designed are the same, only one of the TIFY genes (JrTIFY10) was used in the experiment. Gene-specific primers were given in Table S1. A total of 36 TIFY genes of two varieties were amplified respectively by PCR. Then qRT-PCR (quantitative real-time PCR) was used to measure $20 \mu \mathrm{L}$ reaction mixture with TB Green ${ }^{\text {TM }}$ Premix Ex TaqTMII (TaKaRa, Xi'an, Shaanxi, China) on Bio-Rad CFX ManagerTM Software3.1 in triplicate. The reaction procedure was as follows: $95^{\circ} \mathrm{C}, 30 \mathrm{~s}$ pre denaturation; $95^{\circ} \mathrm{C}, 5 \mathrm{~s}$ denaturation; annealing to $58^{\circ} \mathrm{C}, 30 \mathrm{~s}$, then heating to $72^{\circ} \mathrm{C} 30 \mathrm{~s}, 40$ cycles, the last $95^{\circ} \mathrm{C}, 10 \mathrm{~s}$. The qRT-PCR for each gene had three replicates [28]. The 18S-RNA gene was used as a control [55]. The data was analyzed and processed. The relative expression was calculated as $2^{-\triangle \triangle C t}$ [56]. The experimental details were shown in Table S2.

\section{Abbreviations}

TIFY: TIF[F/Y]XG; JAZ: Jasmonate ZIM-Domain; PPD: PEAPOD proteins; ZML: ZIM-LIKE proteins; ZIM: Zinc-finger protein expressed in Inflorescence Meristem; qRT-PCR: Quantitative real time polymerase chain reaction; DBD: DNA-binding domain; FAO: The Food and Agriculture Organization; JA: Jasmonate acid; GATA: (A/T)GATA(A/G) sequence; TFs: Transcription factors; COl1: CORONATINE INSENSITIVE 1; SA: Salicylic acid; ET: Ethylene; ABA: Abscisic acid.

\section{Supplementary Information}

The online version contains supplementary material available at https://doi. org/10.1186/s12864-022-08416-9.

\section{Additional file 1. \\ Additional file 2. \\ Additional file 3. \\ Additional file 4. \\ Additional file 5 .}

\section{Acknowledgements} Not applicable.

\section{Authors' contributions}

Conceptualization, SBP; methodology, XJL; investigation, FYY; resources, SBP; data curation, FYY; visualization, GYY and XQL; writing —original draft preparation, $X J L$ and FYY; writing - review and editing, SBP. All authors have read and agreed to the published version of the manuscript.

\section{Funding}

This research was funded by the National Natural Science Foundation of China (grant number "31670650"), the Ministry of Science and Technology of the People's Republic of China (grant number "2017YFD0600103-4-2") and Northwest A \& F University (grant number "TGZX2020-05"). The funding agency was not involved in the design of the study, collection, analysis, interpretation of data and writing the manuscript.

\section{Availability of data and materials}

All data generated or analyzed during this study are included in this published article [and its supplementary information files]. The domain architecture of the TIFY genes was obtained from Pfam database (http://pfam.sanger.ac.uk). The chromosomal locations in the walnut genome (J. regia) were downloaded from NCBI database (https://doi.org/10.1093/gigascience/giaa050). The walnut TIFY gene sequences were downloaded from iTAK (http://itak.feilab. net/cgi-bin/itak/index.cgi) database. ExPASy proteomics server (https://web. expasy.org/protparam/) was used to estimate the amino acid number, the molecular weight and the theoretical isoelectric point (PI). The MEME tools (http://meme-suite.org/tools/meme) was chosen to analyze the conservative motifs. The WoLF PSORT (https://www.genscript.com/wolf-psort.html) was used to analyze subcellular localization. The PlantCARE (http://bioinformatics. psb.ugent.be/webtools/plantcare/htm//) was used to predict the cis-acting elements in the promoter region.

\section{Declarations}

\section{Ethics approval and consent to participate}

The 'Qingxiang' and 'Xiangling' walnut were grown and collected in the greenhouse located in Northwest A\&F University (Yangling, Shaanxi province, China), and all samples from these cultivar was adopted for all experiment. These plant materials don't include any wild species at risk of extinction. No specific permits are required for sample collection in this study. We comply with the IUCN Policy Statement on Research Involving Species at Risk of Extinction and the Convention on the Trade in Endangered Species of Wild Fauna and Flora.

\section{Consent for publication}

Not applicable.

\section{Competing interests}

The authors declare that they have no competing interests.

\section{Author details}

${ }^{1}$ Laboratory of Walnut Research Center, College of Forestry, Northwest A \& F University, Yangling 712100, Shaanxi, China. ${ }^{2}$ Key Laboratory of Bio-Resource and Eco-Environment of Ministry of Education, College of Life Science, Sichuan University, Chengdu 610065, Sichuan, China. ${ }^{3}$ Department of Foreign Languages, Northwest A \& F University, Yangling 712100, Shaanxi, China.

Received: 7 October 2021 Accepted: 21 February 2022

Published online: 07 March 2022

\section{References}

1. Kasmi M, Petrit RP, Hodaj B, Kukali E, Rabeta A. Budding of walnut (Juglans regia L.). Albanian J Agri Sci. 2013;12(3):465-9.

2. Sun YD, Yang GY, Jia CX, Hu D, Zhai MZ. Gene identification and functional analysis of temperature stress response in Walnut JRHSP20-1. J Southwest China Normal Univ (Natural Science Edition). 2016;231:95-100.

3. Behrooz A, Vahdati K, Rejali F, Lotfi M, Sarikhani S, Leslie CA. Arbuscular mycorrhiza and plant growth-promoting bacteria alleviate drought stress in walnut. HortScience. 2019;54(6):1087-92. 
4. Zhang Q, Yao YB, Li YH, Luo ZX, Zhang CJ, Li DL, et al. Research progress and prospect of drought meteorological disaster monitoring and early warning and mitigation technology in northwest China. Adv Earth Sci. 2015;243:14-31.

5. Bian RR, Sun ZJ, Li XH, Li HJ. The present situation and prospect of the technology of saline-alkali land improvement and utilization in Northwest China. Ningxia Eng Technol. 2013;64:122-6.

6. Vahdati K, Lotfi N, Kholdebarin B, Hasani D, Amiri R. Screening for drought-tolerant genotypes of Persian walnuts (Juglans regia $L$ ) during seed germination. HortScience. 2009;44(7):1815-9.

7. Shikata M, Matsuda Y, Ando K, Nishii A, Takemura M, Yokota A, et al. Characterization of Arabidopsis ZIM, a member of a novel plant-specific GATA factor gene family. J Exp Bot. 2004;55(397):631-9.

8. Staswick PE. JAZing up jasmonate signaling. Trends Plant Sci. 2008;13(2):66-71.

9. Chung HS, Niu YJ, Browse J, Howe GA. Top hits in contemporary JAZ: an update on jasmonate signaling. Phytochemistry. 2009;70(13-14):1547-59.

10. Wang Y, Pan F, Chen DM, Chu WY, Liu HL, Xiang Y. Genome-wide identification and analysis of the Populus trichocarpa TIFY gene family. Plant Physiol Biochem. 2017;115:360-71.

11. Shi M, Zhou W, Zhang J, Huang S, Wang H, Kai G. Methyl jasmonate induction of tanshinone biosynthesis in Salvia miltiorrhiza hairy roots is mediated by JASMONATE ZIM-DOMAIN repressor proteins. Sci Rep. 2016;6:20919.

12. Melotto M, Mecey C, Niu Y, Chung HS, Katsir L, Yao J, et al. A critical role of two positively charged amino acids in the Jas motif of Arabidopsis JAZ proteins in mediating coronatine- and jasmonoyl isoleucine-dependent interactions with the COI1 F-box protein. Plant J. 2008;55:979-88.

13. Chung HS, Howe GA. A critical role for the TIFY motif in repression of jasmonate signaling by a stabilized splice variant of the JASMONATEZIMdomain protein JAZ10 in Arabidopsis. Plant Cell. 2009:21(1):131-45.

14. Chini A, Fonseca S, Chico JM, Fernández-Calvo P, Solano R. The ZIM domain mediates homo- and heteromeric interactions between Arabidopsis JAZ proteins. Plant J. 2009;59(1):77-87.

15. Chini A, Fonseca S, Fernández G, Adie B, Chico JM, Lorenzo O, et al. The JAZ family of repressors is the missing link in jasmonate signalling. Nature. 2007:448:666-71.

16. Thines B, Katsir L, Melotto M, Niu YJ, Mandaokar A, Liu GH, et al. JAZ repressor proteins are targets of the SCF ${ }^{\text {COIl }}$ complex during jasmonate signalling. Nature. 2007;448:661-5.

17. Zhang M, Chen Y, Nie L, Jin XF, Fu CH, Yu LJ. Molecular, structural, and phylogenetic analyses of Taxus chinensis JAZs. Gene. 2017;620:66-74.

18. White DW. PEAPOD regulates lamina size and curvature in Arabidopsis. Proc Natl Acad Sci USA. 2006;103:13238-43.

19. Heidari P, Faraji S, Ahmadizadeh M, Ahmar S, Mora-Poblete F. New Insights Into Structure and Function of TIFY Genes in Zea mays and Solanum lycopersicum A Genome-Wide Comprehensive Analysis[J]. Front Genet. 2021;12:657970

20. Wasternack C, Hause B. Jasmonates: biosynthesis, perception, signal transduction and action in plant stress response, growth and development. Ann Bot. 2013:111:1021-58.

21. Manfield IW, Devlin PF, Jen CH, Westhead DR, Gilmartin PM. Conservation, Convergence, and divergence of light-responsive, circadian-regulated, and tissue-specific expression patterns during evolution of the Arabidopsis GATA gene family. Plant Physiol. 2007;143:941-58.

22. Lacatus G, Sunter G. The Arabidopsis PEAPOD2 transcription factor interacts with geminivirus AL2 protein and the coat protein promoter. Virology. 2009;392:196-202.

23. Barkoulas M, Galinha C, Grigg SP, Tsiantis M. From genes to shape: regulatory interactions in leaf development. Curr Opin Plant Biol. 2007;620:66-74.

24. Huang $X Z$, Zeng $X F$, Li JR, Zhao DG. Creation and analysis of rice transcription factors TIFY1A and TIFY1B mutants based on CRISPR/Cas9 technology. J Agri Biotechnol. 2017;25(06):1003-12.

25. Ye HY, Du H, Tang N, Li XH, Xiong LZ. Identification and expression profiling analysis of TIFY family genes involved in stress and phytohormone responses in rice. Plant Mol Biol. 2009;71:291-305.

26. Zhao CY, Pan XW, Yu Y, Zhu YM, Kong FJ, Sun $X$, et al. Overexpression of a TIFY family gene, GSJAZ2, exhibits enhanced tolerance to alkaline stress in soybean. Mol Breeding. 2020;40:33.
27. Hu LZ, Wang JS, Zhao JH, Wang QX, Han JY, Ji XE. Identification and comparative analysis of TIFY family genes between tomato and potato. Molecular plant breeding. 2017;15:26-37.

28. Liu XJ, Meng PP, Yang GY, Zhang MY, Peng SB, Zhai MZ. Genome-wide identification and transcript profiles of walnut heat stress transcription factor involved in abiotic stress. BMC Genomics. 2020;21:474.

29. Ma YL, Cao J, He JH, Chen QQ, Li XF, Yang L. Molecular mechanism for the regulation of $\mathrm{ABA}$ homeostasis during plant development and stress responses. Int J Mole Sci. 2018;19:3643.

30. Berens ML, Berry HM, Mine A, Argueso CT. Tsuda K. Evolution of Hormone Signaling Networks in Plant Defense. Annu Rev Phytopathol. 2017:55:401-25.

31. Nathan DG, Azra G, Sofie G, Alain G. Transcriptional machineries in jasmonate-elicited plant secondary metabolism. Trends Plant Sci. 2012;17(6):349-59.

32. Wen D, Wang MY, Mi YL, Ma W, Sun W, Shi YH. Identification and functional analysis of TIFY gene family of hemp (Cannabis sativa L). Chin J Exp Tradit Med Formulae. 2020;26(24):134-43.

33. Zhao XX, Xie KL, Zhang SM, Zhang C, Xi YJ, Sun FL. Identification and analysis of TIFY gene family in switchgrass. Acta Agrestia Sinica. 2019;27(05):1126-37.

34. Zhang Y, Gao M, Singer SD, Fei Z, Wang H, et al. Genome-Wide Identification and Analysis of the TIFY Gene Family in Grape. PLOS ONE. 2012;7(9):e44465

35. Yang, Y, Ahammed GJ, Wan C, Liu H, Zhou Y. Comprehensive Analysis of TIFY Transcription Factors and Their Expression Profiles under Jasmonic Acid and Abiotic Stresses in Watermelon. Int J Genomics.2019;2019:6813086.

36. Li L, Liu YC, Huang Y, Li B, Ma W, Wang DH, et al. Genome-Wide Identification of the TIFY Family in Salvia miltiorrhiza Reveals That SmJAZ3 Interacts With SmWD40-170, a Relevant Protein That Modulates Secondary Metabolism and Development. Front Plant Sci. 2021;12:630424.

37. Lotfi N, Soleimani A, Vahdati K, Çakmakçı R. Comprehensive biochemical insights into the seed germination of walnut under drought stress. Sci Hortic. 2019;250:329-43.

38. Zhang X, Ran W, Zhang J, Ye M, Lin S, Li X, et al. Genome-Wide identification of the tify gene family and their expression profiles in response to biotic and abiotic stresses in tea plants (Camellia sinensis). Int J Mole Sci. 2020;21:8316

39. An XH, Hao YJ, Li EM, Xu K, Cheng CG. Functional identification of apple MdJAZ2 in Arabidopsis with reduced JA-sensitivity and increased stress tolerance. Plant Cell Rep. 2017;36(2):255-65.

40. Xie SF, Cui LC, Lei XL, Yang G, Li J, Nie XJ, et al. The TIFY gene family in wheat and its progenitors: Genome-wide identification, evolution and expression analysis. Curr Genomics. 2019;20(5):371-88.

41. He X, Kang Y, Li WQ, Liu W, Xie P, Liao L, et al. Genome-wide identification and functional analysis of the TIFY gene family in the response to multiple stresses in Brassica napus L. BMC Genomics. 2020;21:736.

42. Wang H, Leng X, Xu X, Li C. Comprehensive analysis of the TIFY gene family and its expression profiles under phytohormone treatment and abiotic stresses in roots of Populus trichocarpa. Forests. 2020;11:315.

43. Pauwels L, Ritter A, Goossens J, Durand AN, Liu HX, Gu YN, et al. The ring E3 ligase keep on going modulates JASMONATE ZIM-DOMAIN12 stability. Plant Physiol. 2015;169(2):1405-17.

44. Liu X, Zhao C, Yang L, Zhang Y, Wang Y, Fang Z, et al. Genome-Wide Identification, Expression Profile of the TIFY Gene Family in Brassica oleracea var capitata, and Their Divergent Response to Various Pathogen Infections and Phytohormone Treatments. Genes. 2020;11:127.

45. Li D, Liu P, Yu J, Wang L, Dossa K, Zhang Y, et al. Genome-wide analysis of WRKY gene family in the sesame genome and identification of the WRKY genes involved in responses to abiotic stresses. BMC Plant Biol. 2017;17:152.

46. Chen X, Chen R, Wang Y, Wu C, Huang J. Genome-Wide identification of WRKY transcription factors in Chinese jujube (Ziziphus jujube Mill) and their involvement in fruit developing, ripening, and abiotic stress. Genes. 2019;10:360.

47. Annarita M, Monica B, Paulo AZ, Aleksey VZ, Rachael EW, Daniela P, et al. High-quality chromosome-scale assembly of the walnut (Juglans regia L) reference genome[J]. GigaScience. 2020;9:5.

48. Finn RD, Mistry J, Tate J, Coggill P, Heger A, Pollington JE, et al. The Pfam protein families database. Nucleic Acids Res. 2010;40(264):263-6. 
49. Camacho C, Coulouris G, Avagyan V, Ma N, Papadopoulos J, Bealer K, et al. BLAST+: architecture and applications. BMC Bioinformatics. 2009;10:421.

50. Xie SY, Zhou CZ, Zhu C, Zhan DM, Chen L, Wu ZM, et al. Genome-wide identification and expression analysis of CSTIFY transcription factor family under abiotic stress and hormone treatment in Camellia sinensis. Acta Horticulturae Sinica. 2021;49(1):1-18.

51 Larkin MA, Blackshields G, Brown NP, Chenna R, McGettigan PA, McWilliam H, et al. Clustal W and Clustal X version 20. Bioinformatics. 2007:23:2947-8.

52. Tamura K, Stecher G, Peterson D, Filipski A, Kumar S. MEGA6 Molecular Evolutionary Genetics Analysis Version 60 . Mole Biol Evol. 2013;30(12):2725-9.

53. Bailey TL, Boden M, Buske FA, Frith M, Grant CE, Clementi L, et al. MEME SUITE: tools for motif discovery and searching. Nucleic Acids Res. 2009;37:W202-8.

54. Liu XJ, Hong HB, Li M, Liu Y, Yang KQ. Extraction of Walnut TOTAL RNA by modified CTAB method. Shandong Agricultural Sciences. 2008;01:97-9.

55. Yang G, Zhang W, Liu Z, Yi-Maer AY, Zhai M, Xu Z. Both JrWRKYZ and JrWRKY7 of Juglans regia mediate responses to abiotic stresses and abscisic acid through formation of homodimers and interaction. Plant Biol. 2017;19(2):268-78.

56. Quan SW, Niu JX, Zhou L, Xu H, Ma L, Qin Y. Identification and characterization of NF-Y gene family in walnut (Juglans regia L). BMC Plant Biol. 2018;18:255.

\section{Publisher's Note}

Springer Nature remains neutral with regard to jurisdictional claims in published maps and institutional affiliations.

- fast, convenient online submission

- thorough peer review by experienced researchers in your field

- rapid publication on acceptance

- support for research data, including large and complex data types

- gold Open Access which fosters wider collaboration and increased citations

- maximum visibility for your research: over $100 \mathrm{M}$ website views per year

At BMC, research is always in progress.

Learn more biomedcentral.com/submissions 